\title{
Learning curve in laparoscopic hysterectomy: yuva experience
}

\author{
Anshu Sharma, Priyanka Chaudhary, Prachi Singhal, Rajiv Acharya, Alok Sharma, \\ Vineeta Gupta, Bhawna Sharma
}

\begin{abstract}
Corresponding author: Dr Priyanka Chaudhary, Associate Professor, Department of Obstetrics and Gynaecology, Shri Guru Ram Rai Institute of Medical and Health Sciences, Patel Nagar, Dehradun, Uttrakhand, India; Email - Dishitapriya07@gmail.com
\end{abstract}

Distributed under Attribution-Non Commercial - Share Alike 4.0 International (CC BY-NC-SA 4.0)

\begin{abstract}
Objective: To evaluate the learning process for laparoscopic hysterectomy ( $\mathrm{LH}$ ) among yuva surgeons. Material and methods: A retrospective comparative study was conducted of all laparoscopic hysterectomies by yuva surgeons (<40years) at Shri Guru Ram Rai Medical college, Dehradun. All the cases of laparoscopic hysterectomy from April 2016 to March 2018 were included in the study. The cases done from April 2016 to November 2016 were categorised in group A, from December 2016 to July 2017 were categorised in group B and from August 2017 to March 2018 were categorised in group C. Various parameters including number of cases, time period, laparotomy conversion, complications and duration of hospital stay were studied. Results: Laparoscopic hysterectomy was performed in 92 patients, out of which 16 cases were in group A, 31 in group B and 45 cases in group $\mathrm{C}$. The mean duration of the operation was 160 minutes in group A,124 minutes in group B and 140 minutes in group C. Average length of hospital stay was 5 days in group A and 4 days in group B and C. The reason for converting to laparotomy in 17 cases (6 in group A, 7 in group B and 4 in group C) was achieving hemostasis (in 7 cases), dense adhesion due to endometriosis ( in 4 cases) and obliteration the pouch of Douglous by large myoma (in 6 cases). Ureteric injury occurred in 2 cases ( 1 case managed intraoperatively another diagnosed after a fortnight and managed thereafter). Bladder injury detected and managed intraoperatively in one case. Conclusion: Minimal invasive surgery has been accepted as a new appropriate option, replacing open surgical technique in almost all surgical disciplines. To reach to an optimal level of surgical skill adequate training, number of cases and time is required. However, route of surgery should be judiciously decided after careful case selection.
\end{abstract}

Keywords: Laparoscopic hysterectomy, learning curve, bladder injury, vaginal hysterectomy.

Hysterectomy is one of the commonest surgeries performed globally. Various routes of performing hysterectomy include abdominal, vaginal and laparoscopic. History of laparoscopic hysterectomy dates back to 1984 when Kurt Semm in Germany performed the first laparoscopic assisted vaginal hysterectomy (LAVH). Harry Reich is credited to have revolutionized the world of gynaecological surgery by performing the first total laparoscopic hysterectomy (TLH) in January 1988 in Pennsylvania. ${ }^{1}$ Laparoscopic hysterectomy ( $\left.\mathrm{LH}\right)$ is divided into three main subgroups: laparoscopically assisted vaginal hysterectomy(LAVH) where vaginal hysterectomy is preceded by laparoscopic procedures excluding uterine artery ligation; laparoscopic hysterectomy where the laparoscopic

Received: $29^{\text {th }}$ February 2020. Accepted: $4^{\text {th }}$ April 2020.

Sharma A, Chaudhary P, Singhal P, Acharya R, Sharma A, Gupta V, et al. Learning curve in laparoscopic hysterectomy: yuva experience. The New Indian Journal of OBGYN. 2020; 7(1): 48-51. 
procedures include uterine artery ligation $[\mathrm{LH}(\mathrm{a})]$; and total laparoscopic hysterectomy (TLH) where a vaginal component is absent and the vaginal vault is sutured laparoscopically. ${ }^{2}$

Both minimally invasive hysterectomies and vaginal hysterectomies are associated with reduced blood loss and hospital stay, lower incidence of sepsis, faster return to routine activity resulting in a greater degree of patient satisfaction when compared to more traditional route of hysterectomy, total abdominal hysterectomy (TAH). ${ }^{3}$ Various studies have demonstrated a number of advantages of laparoscopic hysterectomy. Despite the absolute advantages of minimal invasive hysterectomy over its abdominal counterpart, the latter remains the commonest approach for hysterectomy. ${ }^{4}$ The present study was done to observe the learning pattern of laparoscopic hysterectomy amongst young surgeons keen on acquiring skills to choose the laparoscopic routes.

\section{Material and methods}

It was a retrospective comparative study of all laparoscopic hysterectomies by surgeons $<40$ years at Shri Guru Ram Rai Medical College, Dehradun. All cases of laparoscopic hysterectomy from April 2016 to March 2018 were included in the study. The cases operated from April 2016 to November 2016 were categorised in group A, from December 2016 to July 2017 were categorised in group B and from August 2017 to March 2018 were categorised in group C. All the surgeries i.e laparoscopic assisted vaginal hysterectomy, laparoscopic hysterectomy and total laparoscopic hysterectomy were included in the study Various parameters including number of cases, time period, conversion to laparotomy, complications and duration of hospital stay were studied. The cases which were done by experts in laparoscopic workshops or cases where a senior consultant had to intervene were excluded from the study.

\section{Results}

There were a total of 239 hysterectomies for gynaecological indications performed by surgeons who were younger than 40 years of age. Of these, total abdominal hysterectomies were 108, non descent vaginal hysterectomies were 39 and Laparoscopic hysterectomy was performed in 92 patients. In group $\mathrm{A}$, the most common route of hysterectomy was total abdominal hysterectomy $(60.7 \%$ i.e 72 out of 108 cases) and laparoscopic hysterectomy was done in $19.3 \%$ (30) cases only. In group $\mathrm{B}$, the laparoscopic hysterectomy had increased to $39.3 \%$ (52 out of 133 cases) with a subsequent fall in the total laparoscopic hysterectomy i.e.43\% (65) cases. A similar trend continued in group $\mathrm{C}$ when laparoscopic approach became the most common route of hysterectomy i.e $59.2 \%$ (69 cases) and total abdominal hysterectomy was done only in $30.3 \%$ (49 cases). This rise in laparoscopic hysterectomy was statistically significant $(p<0.01)$. Simultaneously a decline in nondescent vaginal hysterectomy was observed from $20.0 \%$ (26 cases) in group A to $10.5 \%$ (18 cases) in group $\mathrm{C}$.

Table 1 shows the various parameters related to the learning process of the surgery over a period of time. The
Table 1: Comparison of various operative parameters in 3 groups

\begin{tabular}{llll}
\hline Parameters & Group A & Group B & Group C \\
\hline No. of cases & 16 & 31 & 45 \\
Time (in minutes) & 160 & 124 & 140 \\
Opening & 6 & 7 & 4 \\
Complication & 1 & 2 & 1 \\
\hline
\end{tabular}

mean duration of the operation was 160 minutes in group $\mathrm{A}$ which decreased to 124 minutes in group $\mathrm{B}$ and then again increased to 140 minutes in group C. In group A vaginal vault closure was done vaginally in all the cases. In group B, 9 out of 34 vaginal vaults $(29 \%)$ were closed by endosuturing and in group $\mathrm{C}$ in a majority of cases (34 out of 45 i.e $75.5 \%$ ) endosuturing of vaginal vault was performed. This led to increased mean duration of surgery in group $\mathrm{C}$ as compared to Group B.

In a few cases amongst all groups, the procedure was converted to laparotomy. The reason for conversion in 17 cases ( 6 in group A, 7 in group B and 4 in group C) was to achieve satisfactory hemostasis in 6 cases, presence of dense adhesion in 4 cases and obliteration of the pouch of Douglas by large myoma (in 4 cases) or technical failure like problem in $\mathrm{CO}_{2}$ insufflation or failure of energy source (in 3 cases). With the passage of time a decline in conversion to laparotomy was observed from $37.5 \%$ in group A to $8.9 \%$ in group $\mathrm{C}$; the difference being statistically significant $(\mathrm{p}<0.05)$.

The rate of complications also declined as expertise increased. In group A, there was one ureteric injury that was identified intraoperatively while coagulating the left uterosacral ligament and was managed intraoperatively. Post operative period was uneventful. In group B, there was one right ureteric injury which was identified after a fortnight when the patient complained of copious vaginal discharge of 3 days duration. On cystoscopy, the ureter was found to be necrosed at uretrovesical junction. Percutaneous nephrostomy followed by reanastomosis of ureter to the 
bladder was done by the urologist. On follow up the patient was recovered completely. In group $\mathrm{C}$, there was one bladder injury that was identified during routine cystoscopy after endosuturing of the vault; repaired by urologist at the same time. There were no bowel and vascular injuries in any group. At the end of the study a questionnaire was given to all the yuva surgeons to elaborate their experience in laparascopy (Table 2). Most surgeons believed that over a were sutured by endosuturing that led to an increased duration of surgery in group $\mathrm{C}$ compared to group B.

American College of Obstetricians and Gynecologists has recommended vaginal hysterectomy as the optimum route for surgery and when hysterectomy by vaginal route is not possible minimal invasive surgery should be preferred over abdominal hysterectomy. ${ }^{7}$ In the famous study done by Brummer et al in Finland from 2000-2005 it was found that

Table 2: Questionnaire answered by yuva surgeons (doing laparoscopic hysterectomies)

\begin{tabular}{|c|c|c|c|}
\hline Questions & Group A & Group B & Group C \\
\hline Most common route of hysterectomy & $\mathrm{TAH}$ & $\mathrm{TAH}$ & TLH \\
\hline Most difficult step & Uterine artery ligation & Vault opening & Endosuturing \\
\hline Most rate limiting step & Bladder dissection & Uterine artery coagulation & Endosuturing \\
\hline $\begin{array}{l}\text { Most common complication you } \\
\text { anticipate before surgery }\end{array}$ & Bladder injury & Ureter and bladder injury & Ureter injury \\
\hline Can do without supervision & No & No & May be / yes \\
\hline $\begin{array}{l}\text { Measures that can further help in } \\
\text { improvement }\end{array}$ & None & None & Consistent performance \\
\hline
\end{tabular}

TAH $=$ Total abdominal hysterectomy, TLH = Total laparoscopic hysterectomy

period of time their skills had improved. Although they felt confident about performing the surgery independently, a fear of ureteric and bladder injury always remained in mind.

\section{Discussion}

Minimally invasive surgery has been steadily replacing the open standard technique in several procedures across various surgical disciplines. The concept of learning curve in surgical field was adopted in 1989 with the advent of minimal invasive surgery. Learning a skill follows a typical pattern of initial difficulty and increased complications followed by ease of performing a procedure and decline in the rate of complication and time taken to perform the procedure followed by stabilization of performance in terms of speed, recovery and complications. ${ }^{4}$ In our study a steady improvement could be appreciated in laparoscopic hysterectomy from group A till $\mathrm{C}$ in terms of increased number of cases, decline in the perioperative complication rate, need of conversion to laparotomy, hospital stay and improved recovery. The results are consistent with various studies performed on learning curve of laparoscopic hysterectomy. ${ }^{5,6}$ However, the duration of surgery did not follow the usual pattern of steady decline from group A to group $\mathrm{C}$. This is because in group A, vaginal vaults in all the cases were sutured vaginally where as in group B, 29\% of vaults were sutured vaginally and in group $\mathrm{C} 75.6 \%$ vaults in 2000, the proportion of abdominal hysterectomy (AH) was $38 \%$, vaginal hysterectomy ( $\mathrm{VH}) 37 \%$ and $\mathrm{LH} 25 \%$, whereas in 2005 , the proportions were $26 \%, 45 \%$ and $29 \%$, respectively. ${ }^{8}$ In our study the total number of hysterectomy remained constant in the past two years. Similar to the above mentioned study, total abdominal hysterectomy decreased to half over this period of time and laparoscopic hysterectomy increased three fold. However, a disturbing fact was observed that non descent vaginal hysterectomy has decreased to half in past two years. The reasons behind the decline in utilization of natural orifice solely for the purpose of uterus removal can be associated adnexal mass, endometriosis, pelvic inflammatory disease, previous caesareans or other abdominal surgery leading to suspicion of adhesions and poor vaginal descent that makes the vaginal surgery difficult. The other reason could be a lack of opportunity of formal training in vaginal hysterectomy as compared to laparoscopic hysterectomy as the latter is easier to demonstrate and learn. Lastly, laparoscopic surgery is considered more exciting and modern leading to an increased inclination of young surgeons towards the minimally invasive technique.

The choice of route of hysterectomy at the end lies in the hands of the operating surgeon and is determined by various factors which include the indication and likely associated 
The New Indian Journal of OBGYN. 2020 (July-December); 7(1)

complications, skill and experience of the surgeon and availability of equipment.

Various studies have concluded that a learning experience of 30 laparoscopic hysterectomies by individual surgeon is essential to reach a desirable level of proficiency and to anlayse the learning curve. ${ }^{5,6}$ Limitations of our study were small sample size, and that the surgeries were performed by single set of surgeons hence it may not be reproducible in other centres. There is a felt need of a larger randomized study before concluding the minimum number of cases required to be performed to attain optimal outcomes.

\section{Conclusion}

Minimal invasive surgery has been replacing the open surgical technique in almost all surgical disciplines. To reach to an optimal standard of surgical skill adequate training, number of cases and time is required. However, route of surgery should be judiciously decided after careful case selection.

\section{Conflict of interest: None. Disclaimer: Nil.}

\section{References}

1. Reich H, De Caprio J, Mc Glynn F. Laparoscopic hysterectomy. J Gynecol Surg. 1989; 5: 213-6.

2. Johnson N, Barlow D, Lethaby A, Tavender E, Curr E, Garry R. Surgical approach to hysterectomy for benign gynaecological disease. Cochrane Database Sys Rev. 2006; 2: CD003677.

3. Kumar P, Swain S. Diagnostic and therapeutic laparoscopy in the management of endometriosis. Int $\mathrm{J}$
Reprod Contracept Obstet Gynecol. 2018; 7(11): 46954700

4. Wu JM, Wechter ME, Geller EJ. Hysterectomy rates in the United States, 2003. Obstet Gynecol. 2007; 110:1091-5

5. Altgassen C, Michels W, Schneider A. Learning laparoscopic-assisted hysterectomy. Obstet Gynecol. 2004; 104(2): 308-13.

6. Makinen J, Johansson J, Tomas C. Morbidity of 10110 hysterectomies by type of approach. Human Reprod. 2001; 16(7):1473-8.

7. Choosing the route of hysterectomy for benign disease. ACOG Committee Opinion 444. Obstet Gynecol. 2009; 144: 1156-8.

8. Brummer TH, Seppala TT, Harkki PSM. National learning curve for laparoscopic hysterectomy and trends in hysterectomy in Finland 2000-2005. Human Reproduction. 2008. 23(4); 840-5.

Anshu Sharma ${ }^{1}$, Priyanka Chaudhary ${ }^{2}$, Prachi Singhal ${ }^{3}$, Rajiv Acharya ${ }^{4}$, Alok Sharma ${ }^{5}$, Vineeta Gupta ${ }^{6}$, Bhawna Sharma ${ }^{7}$

${ }^{1}$ Assistant Professor; ${ }^{2}$ Associate Professor; ${ }^{3}$ Assistant Professor; ${ }^{4}$ Professor; ${ }^{6}$ Professor; ${ }^{7}$ Professor, Department of Obstetrics and Gynaecology, Shri Guru Ram Rai Institute of Medical and Health Sciences, Patel Nagar, Dehradun, Uttrakhand, India; ${ }^{5}$ Consultant, Obstetrics and Gynaecology. 